\title{
Photo-ionization models of NGC 2363 and their implications
}

\author{
Valentina Luridiana and Manuel Peimbert \\ Instituto de Astronomía, UNAM, Mexico
}

Claus Leitherer

Space Telescope Science Institute, Baltimore, MD, USA.

\begin{abstract}
We compute photo-ionization models for the giant extragalactic $\mathrm{H}$ II region NGC 2363, and compare them with optical observational data. We focus on the following observational constraints: $F(\mathrm{H} \beta), N_{e}, E W(\mathrm{H} \beta)$, and the ratios of $I(\lambda 5007), I(\lambda 4363), I(\lambda 3727), I(\lambda 6300), I(\lambda 6720)$ and $I(\lambda 4686)$ relative to $I(\mathrm{H} \beta)$. We discuss the variations of the emission spectra obtained with different input parameters. We show that low metallicity models $\left(Z=0.10 \mathrm{Z}_{\odot}\right)$ cannot reproduce the observed features of the spectrum, and that the disagreement can be satisfactorily overcome by allowing for spatial temperature fluctuations in the nebula. Accordingly, we show that the metallicity of NGC 2363 has most probably been underestimated, and that a value of $Z \simeq 0.25 \mathrm{Z}_{\odot}$ is in better agreement with the observational data than the usually adopted value $Z \simeq 0.10 \mathrm{Z}_{\odot}$. We also derive values for the slope and the high mass end of the IMF, as well as the age of the stellar cluster.
\end{abstract}

\section{Introduction}

NGC 2363 is a very luminous giant H II region, located in the south-west end of the irregular galaxy NGC 2366. It is one of the brightest extragalactic H II regions known, with an estimated mass of about $2.2 \times 10^{6} \mathrm{M}_{\odot}$ (Carigi \& Peimbert 1999).

Our aim in the present work is to derive the age, the shape of the star formation law, the IMF and the chemical composition of NGC 2363. This is performed by means of a comparison between photo-ionized models, computed with the photo-ionization code CLOUDY (version 90.04, Ferland 1996), and the observed features of the emission spectrum of the region (González-Delgado et al. 1994; Izotov et al. 1997; Peimbert et al. 1986). To compare the model results with the observed line intensities, we corrected the standard model output for the size of the slit. We assumed that the slit is centered on the region, and considered three different cases with projected size $37 \times 5500 \mathrm{pc}$ (slit A), $22 \times 276 \mathrm{pc}$ (slit B), and $70 \times 228 \mathrm{pc}$ (slit C), corresponding to the slits used by Izotov et al. (1997), González-Delgado et al. (1994, extraction A2), and Peimbert et al. (1986), respectively, under the assumption of a $3.8 \mathrm{Mpc}$ distance to the region.

Our models assume a spherical, hollow, radiation bounded nebula, made up of two concentric shells of different densities. The grid is defined by the following parameters: the star formation law, the initial mass function, the high mass cutoff of the IMF, the age of the cluster, the inner radius of the nebula, 
i.e., the size of the central hole, the electron density, the gas covering factor, the stellar metallicity, and the gas metallicity. Models of NGC 2363 with other geometrical considerations are presented elsewhere (Luridiana 1998).

The ionizing spectra have either been taken from Leitherer et al. (1996), or have been recomputed with the same synthesis code.

\section{Results and conclusions}

Based on the analysis of an extensive grid of photo-ionization models, we suggest that the metal content of NGC 2363 has previously been underestimated. This conclusion is supported by the following facts:

(a) Models with $Z=0.10 \mathrm{Z}_{\odot}$ fail to reproduce the observed emission-line spectrum;

(b) Enhanced metallicity models $\left(Z=0.25 \mathrm{Z}_{\odot}\right)$ reproduce the characteristics of the observed spectrum, with the exception of $I(\lambda 4363)$ and $I(\lambda 6300)$;

(c) There is direct evidence for the presence of WR stars in the region, which supports the $Z=0.25 \mathrm{Z}_{\odot}$ models relative to those with $Z=0.10 \mathrm{Z}_{\odot}$;

(d) WR winds and $\mathrm{SN}$ explosions are expected to generate spatial temperature fluctuations in the nebula, enhancing $I(\lambda 4363)$ and $I(\lambda 6300)$;

(e) Considering the presence of temperature fluctuations, a higher metallicity value is obtained, giving a straightforward explanation for the presence of WR stars in the cluster;

(f) The number of WR stars necessary to account for the observed $\lambda 4686$ flux restricts the range of possible values for the IMF slope and the upper mass limit of the IMF mass $M_{\text {up }}$;

(g) We found a best fit value of 2.00 for the IMF slope, and we estimate that the upper mass limit is about $120 \mathrm{M}_{\odot}$;

(h) The estimated age of the burst is $2.1 \pm 0.5 \mathrm{Myr}$; and

(i) The higher FWHM values for 4686 relative to the main component of the other emission lines support the idea that, in addition to photo-ionization, mechanical energy is also injected to NGC 2363 causing the presence of temperature fluctuations larger than those predicted by the photo-ionization models.

A full discussion of the whole grid of models, supporting these conclusions, will be presented elsewhere (Luridiana et al. 1999).

\section{References}

Carigi, L., Peimbert, M. 1999, in preparation

Izotov, Yu.I., Thuan, T.X., Lipovetski, V.A. 1997, ApJS 108, 1

Ferland, G.J. 1996, HAZY, University of Kentucky Internal Report

González-Delgado, R.M., Pérez, E., Tenorio-Tagle, G. et al. 1994, ApJ 437, 239

Leitherer, C., Alloin, D., Fritze-von Alvensleben, U. et al. 1996, PASP 108, 996

Luridiana, V. 1998, in: D. Friedli, M. Edmunds, C. Robert \& L. Drissen (eds.), Abundance Profiles: Diagnostic Tools for Galaxy History, ASP-CS 147, 138

Luridiana, V., Peimbert, M., Leitherer, C. 1999, in preparation

Peimbert, M., Peña, M., Torres-Peimbert, S. 1986, A\&A 158, 266 